\title{
Panoramic Radiography in the Diagnosis of Carotid Artery Atheromas and the Associated Risk Factors
}

\author{
João César Guimarães Henriques*,1, Eliane Maria Kreich ${ }^{1}$, Márcia Helena Baldani², Mariely Lucia- \\ no $^{2}$, Julio Cezar de Melo Castilho ${ }^{1}$ and Luiz Cesar de Moraes ${ }^{1}$ \\ ${ }^{1}$ School of Dentistry, Universidade Estadual Paulista de São José dos Campos, São Paulo, Brazil \\ ${ }^{2}$ Universidade Estadual de Ponta Grossa, Paraná, Brazil
}

\begin{abstract}
Atherosclerosis is a serious chronic disease, responsible for thousands of deaths worldwide and is characterized by thickening and loss of elasticity of the arterial walls, associated with the presence of atheromatous plaques. Various risk factors act directly on predisposition to the disease, among which the following are pointed out: diabetes mellitus, arterial hypertension and inadequate diet and eating habits. More recent researches have elucidated new risk factors acting in the development of this disease, such as, for example: periodontitis, chronic renal disease and menopause. The panoramic radiograph, commonly used in dental practice, makes it possible to see calcified atherosclerotic plaques that are eventually deposited in the carotid arteries. The aim of this review article was to emphasize the dentist's important role in the detection of carotid artery atheromas in panoramic radiographs and the immediate referral of patients affected by these calcifications to doctors. In addition, the study intended to guide the dentist, especially the dental radiologist, with regard to differential diagnosis, which should be made taking into consideration particularly the triticeal cartilage when it is calcified.
\end{abstract}

Key Words: Atherosclerosis, carotid artery diseases, panoramic radiography, risk factors.

\section{INTRODUCTION}

Atheromas are calcified plaques especially composed of lipids and fibrous tissue, which are deposited on the walls of blood vessels, triggering atherosclerosis. Atherosclerosis in turn, is a chronic inflammatory disease of a multifactorial nature, characterized by thickening and loss of elasticity of the arterial walls, associated with the presence of atheromas. When atherosclerosis affects the arteries that supply the brain, the carotids, strokes can be triggered, and when it affects arteries that supply the heart, the coronary arteries, there is a possibility of myocardial infarction, events that result in the death of thousands of persons all over the world [1].

There is a series of factors predisposing to the development of atherosclerosis, such as: diabetes mellitus, obesity, arterial hypertension, smoking, alcoholism, inadequate diet and eating habits, periodontitis, chronic renal disease, menopause, etc. The atherosclerotic process begins in childhood and the clinical manifestations occur in adult life, particularly after 45 years of age [2].

Panoramic radiographs, commonly used in dentistry, may have a diagnostic function in identifying atheromatous lesions present in the carotid artery. Evaluation and diagnosis of carotid artery atheromatous lesions by means of pano-

\footnotetext{
*Address correspondence to this author at the Avenida Engenheiro Francisco José Longo, $\mathrm{n}^{\circ} 777$ - Bairro: Jardim São Dimas. CEP: 12245-000. Disciplina de Radiologia Odontológica da Faculdade de Odontologia da Universidade Estadual Paulista "Júlio de Mesquita Filho" campus de São José dos Campos- São Paulo, Brazil. Tel: 005512-3947-9054;

Fax: 005512-3947-9000; E-mail: joaocesarhenriques@yahoo.com.br
}

ramic radiographs requires professional acuity and experience, since other radiopaque anatomic structures in the regions may be included in the differential diagnosis, outstanding among them being the triticeal cartilage when it is calcified [3].

The aim of this review article was to emphasize the importance of the panoramic exam as an auxiliary diagnostic method in detecting atheromas of the carotid arteries. Moreover, this study approached the risk factors directly related to the incidence of atheromas in the carotid arteries and the dentist's responsibility to make a diagnosis and refer the patient to a doctor for evaluation with a view to preventing a possible stroke.

\section{REVIEW OF THE LITERATURE}

\section{Etiopathogenesis of Atherosclerosis}

At present, atherosclerotic disease is understood to be an inflammatory disease of an immunological nature. As a result of a probable sum of events, for example, involving metabolic, nutritional, hypertensive abnormalities, and even viral and bacterial infections, the vascular endothelium is injured. Thereby an inflammatory environment is developed and multiple interactions among platelets, $\mathrm{T}$ lymphocytes, macrophages, smooth muscle cells, adhesion molecules and genetic components propagate the pathological condition. Thus, an accumulation of low density lipoproteins (LDL) occurs in the intimate layer of the endothelium, culminating in the formation of atheromatous plaque, which develops with eventual superimposition of calcium deposits [4] (Fig. 1). 


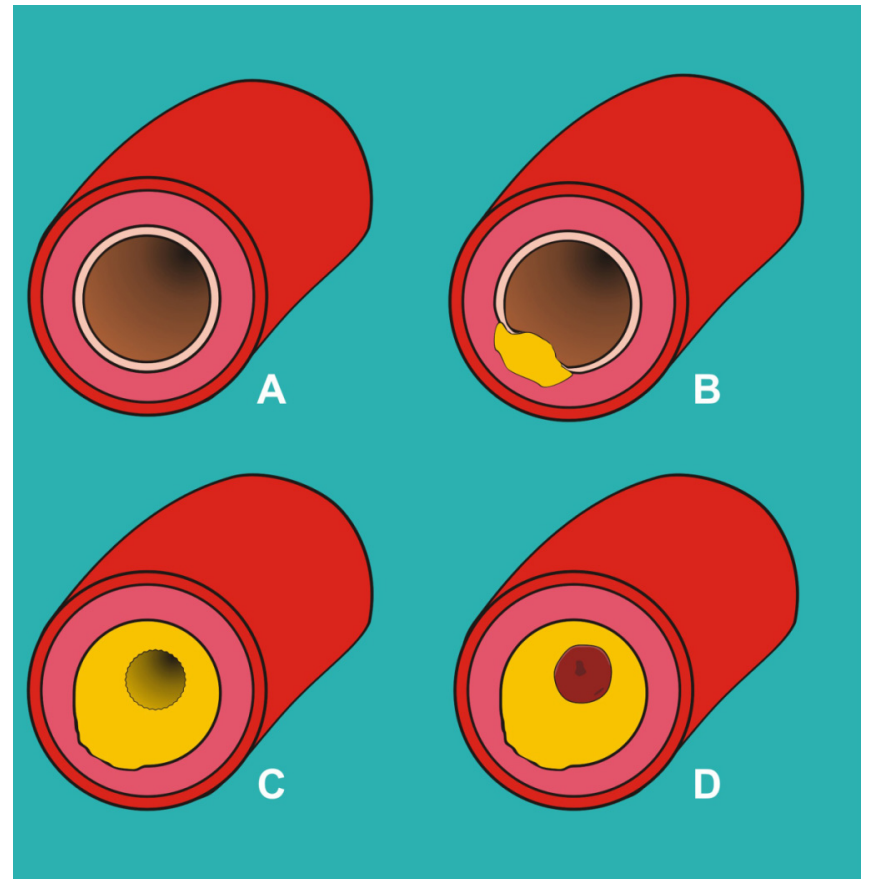

Fig. (1). Simplified diagram of the development of atherosclerosis showing: Cross sectional cut of the artery when it was still whole (A); initial injury of the endothelium (B); the atheromatous plaque formed $(\mathbf{C})$ and a thrombus associated with the plaque, completely obstructing the hollow passage of the vessel (D).

\section{The Risk Factors for Atherosclerosis}

Atherosclerosis is a disease that involves a series of biological events and is associated with different risk factors, especially those listed as follows:

\section{Diabetes Mellitus}

Diabetes mellitus is a complex metabolic disturbance, characterized by chronic hyperglycemia resulting from abnormal insulin secretion. Various complications are involved during the long term course of this pathology, such as: Coronary artery diseases, cerebrovascular diseases, peripheral vascular diseases, difficulty in wound healing and in terms of oral manifestations, xerostomia and greater susceptibility to developing periodontal disease [5].

Panoramic radiographic exams performed in diabetics have revealed the prevalence of carotid artery calcifications in $24 \%$ of the patients treated without insulin and in $36 \%$ of diabetics treated with insulin. These data demonstrate the high risk of stroke in diabetic patients, irrespective of the treatment modality used [6].

\section{Obesity}

Obesity certainly is a world health problem and since atherosclerosis also develops as a result of the accumulation of fatty substances on vascular walls, obese individuals are potential candidates for presenting this disease. Autopsies performed in young persons revealed the presence of atherosclerosis as early as the first decade of life, suggesting that habits normally present in society nowadays, such as childhood sendentarism and the consumption of food with a low nutritional value have determined the increase in obesity parallel to atherosclerotic disease [7].

\section{Arterial Hypertension}

Increased arterial pressure consists of a vascular inflammatory process that in conjunction with atherosclerosis, potentiate the deleterious effects on the endothelium. Scientific evidences have proved the action of endothelial vascular inflammation on both the genesis and the development of arterial hypertension and arteriosclerosis, which contributes to the development of a vicious pathological cycle between the two events [8].

\section{Smoking}

Abandoning the smoking habit reduces the risk of cardiovascular diseases by approximately $50 \%$, and particularly in relation to atherosclerosis, eliminating the smoking habit may reduce eventual risks of death by up to $70 \%$ [9]. Smoking acts by favoring a quantitative increase in bad cholesterol (LDL) and diminishing good cholesterol (HDL). In the blood, smoking may also cause an increase in carbon monoxide, elevating the risks of lesions to the lining of the arterial wall and predisposition to arterial contractions, which reduces the final supply of blood constituents to the tissues even further [10].

\section{Alcoholism}

Patients free of any risk factors for atherosclerosis present very different results in relation to the different degrees of alcohol consumption. A great prevalence of atheromatous plaques was visualized only in patients who consumed large quantities of alcohol. Abstemious individuals and those that consumed small or moderate amounts of alcohol, had equally low prevalence of atherosclerotic disease. Thus, alcohol consumption has been shown to play a very antagonistic role, favoring atherosclerosis; in moderate quantity the substance has a protective action in relation to cardiovascular diseases, whereas in excess it greatly favors its development [11].

\section{Inadequate Diet and Eating Habits}

A diet with high levels of cholesterol favors the progression of cardiovascular diseases, especially stimulating the development of atheromatous plaques. Fatty foods also potentially favor vascular diseases and in this context, fatty acids are divided into two main classes: the saturated types, which act by increasing the total cholesterol and LDL (low density lipoprotein) levels; and the unsaturated fatty acids, subdivided in monounsaturated and polyunsaturated types, which in turn have potentially anti-atherogenic effects, such as the reduction of plasmatic LDL levels, elevation of HDL (high density lipoprotein) levels, increase in vasodilatation and reduction in platelet aggregation [12].

\section{Chronic Renal Disease}

Chronic renal disease has been shown to be associated with the prevalence of atheromas. Panoramic radiographic exams were performed in 50 healthy patients (control group) and in 69 chronic renal patients, of whom 34 were undergoing the hemodialysis process and 35 had been submitted to kidney transplants. Carotid artery calcifications were seen in $17.6 \%$ of the hemodialyzed patients and in $15.7 \%$ of the transplant patients. Whereas in the control group of patients, only $3 \%$ of the individuals presented calcifications [13]. 


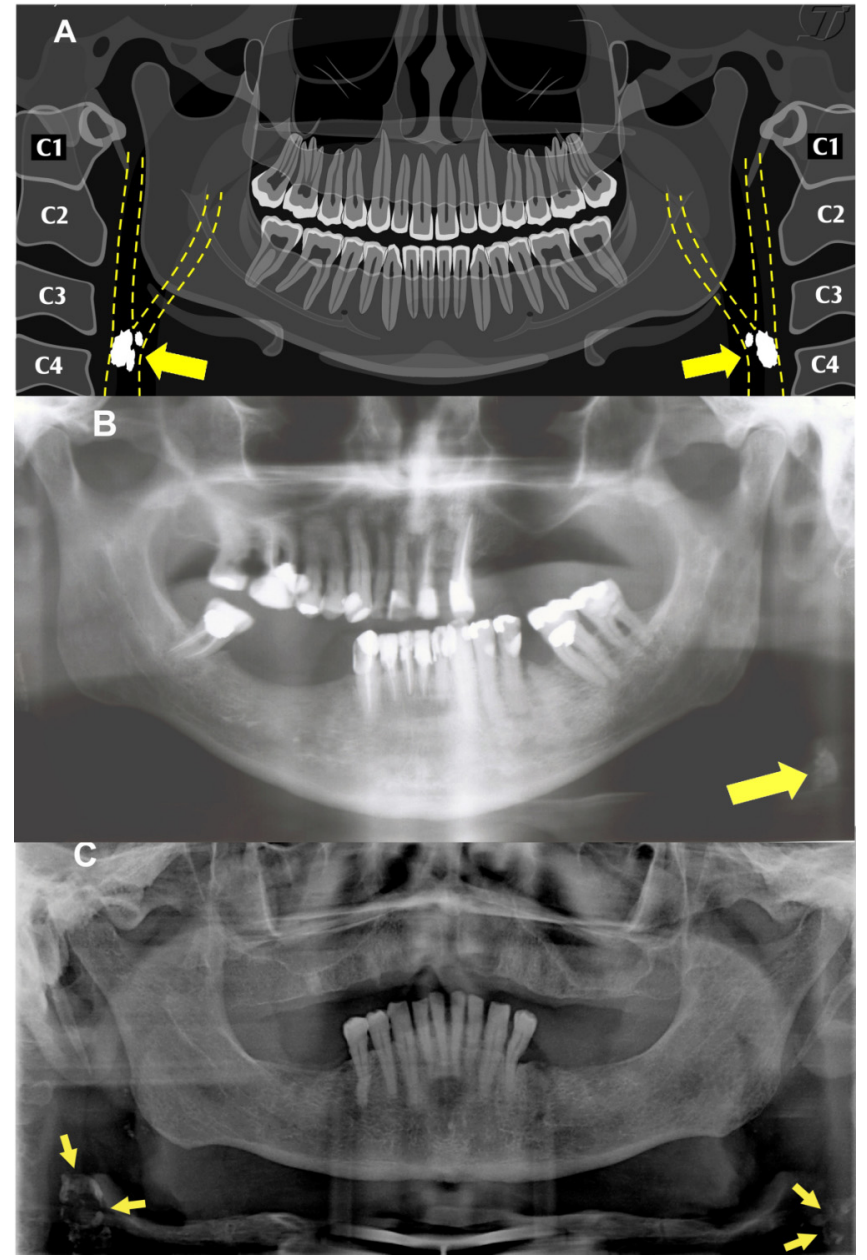

Fig. (2). (A) Diagrammatic illustration of a panoramic radiograph with the arrows indicating the carotid artery atheromas disposed bilaterally. (B) Panoramic radiograph of a patient with an atheroma on the left side. (C) Panoramic radiograph of a chronic renal patient with bilateral atheromas.

\section{Menopause}

The reduced estrogen hormone levels and the other aging processes associated with menopause are factors that increase the stroke risks in women [14]. In view of this, 52 post-menopausal women ranging between 55 and 90 years, were submitted to panoramic radiographic exams that revealed carotid artery calcifications in 16 (31\%) different individuals. Moreover, evaluation of the medical record charts of the women affected by atheromas revealed that $15(94 \%)$ were hypertensive, $4(25 \%)$ were obese and $3(19 \%)$ had diabetes mellitus type II [15].

\section{Other Risk Factors}

In addition to the above-mentioned risk factors, various researches have proved the action of other conditions on predisposition to atherosclerosis, such as: sedentarism, stress, hyperhomocysteinemia, radiotherapy of the head and neck, the obstructive sleep apnea syndrome, aging and being of the male sex. In view of this, it is worth emphasizing that the larger the number of risk factors present and associated, the greater the chance of atherosclerosis occurring [16-22].

\section{Panoramic Radiography and Atheromas in the Carotid Arteries}

Atheromatous plaques, even in cases of partial calcifications, resulting from the deposition of calcium salts, can be observed in panoramic radiographs, which in turn represent one of the imaging exams most requested in dentistry at present. In these radiographs, the image of the atheromas can be presented as one or more irregular radiopacities eventually punctuated by vertical-linear radiolucent areas, with single or multiple affections, of varied sizes, localized approximately $2.5 \mathrm{~cm}$ posterior and inferior to the mandibular angle, adjacent to the space between vertebrae $\mathrm{C} 3$ and $\mathrm{C} 4$, uni- or bilaterally [3,23-25] (Fig. 2).

Panoramic radiography consists of a radiographic method that uses low doses of radiation, is low cost and has technical simplicity that makes it extremely useful in identifying anatomic structures of the head and neck. Moreover, the atheromas are normally deposited along the ascendant trajectory of the common carotid artery that bifurcates into internal and external carotid arteries, and in the absolute majority of cases, is comprised within the area of coverage of the panoramic radiographic cut [26].

The differential diagnosis of the images of carotid artery atheromas in panoramic radiography must be made considering a series of anatomic structures in the cervical and adjacent regions, such as: the hyoid bone, styloid process, triticeal cartilages, thyroid cartilage, epiglottis, calcifications in the stylo-hyoid and stylomandibular ligaments, tonsilloliths, phlebolites, calcified lymphatic nodules, anterior tubercle of the atlas vertebra and even calcified acnes [3].

In the differential diagnosis, emphasis must be laid on the triticeal cartilages, which are localized in an intermediary portion between the hyoid bone and the thyroid cartilage, are of a hyaline nature and have a great propensity to calcification as the individual's age advances. The triticeal cartilages form part of the cartilaginous arsenal of the larynx, are constituted of paired structures and disposed on the posterior free edge of the lateral thyro-hyoid ligaments. The real function of the triticeal cartilages is unknown, although it is believed that they act to strengthen the thyro-hyoid ligament [27]. In the panoramic radiograph, these cartilages present as homogeneous radiopacities when calcified, have regular oval shapes, approximately 2 to $4 \mathrm{~mm}$ wide, 7 to $9 \mathrm{~mm}$ long, usually superimposed on the airspace of the pharynx and are close to the superior portion of vertebra $\mathrm{C} 4$. It is necessary to make a differential diagnosis between the triticeal cartilages and carotid artery atheromas, since these cartilages, differently from atheromas that are a sign that the patient must urgently be referred to a doctor, do not require any treatment [27,28].

Nevertheless, the best way to differentiate atheromas from triticeal cartilages is by considering the fact that the atheromas have a more lateral anatomic localization and the triticeal cartilages a more medial localization. Thus, in the face of doubt arising from analysis of the panoramic radiograph, the indication is that the dentist must request an antero-posterior radiograph. This antero-posterior radiograph must be taken by means of the Modified Towne technique, with the patient's teeth maintained in occlusion and the 


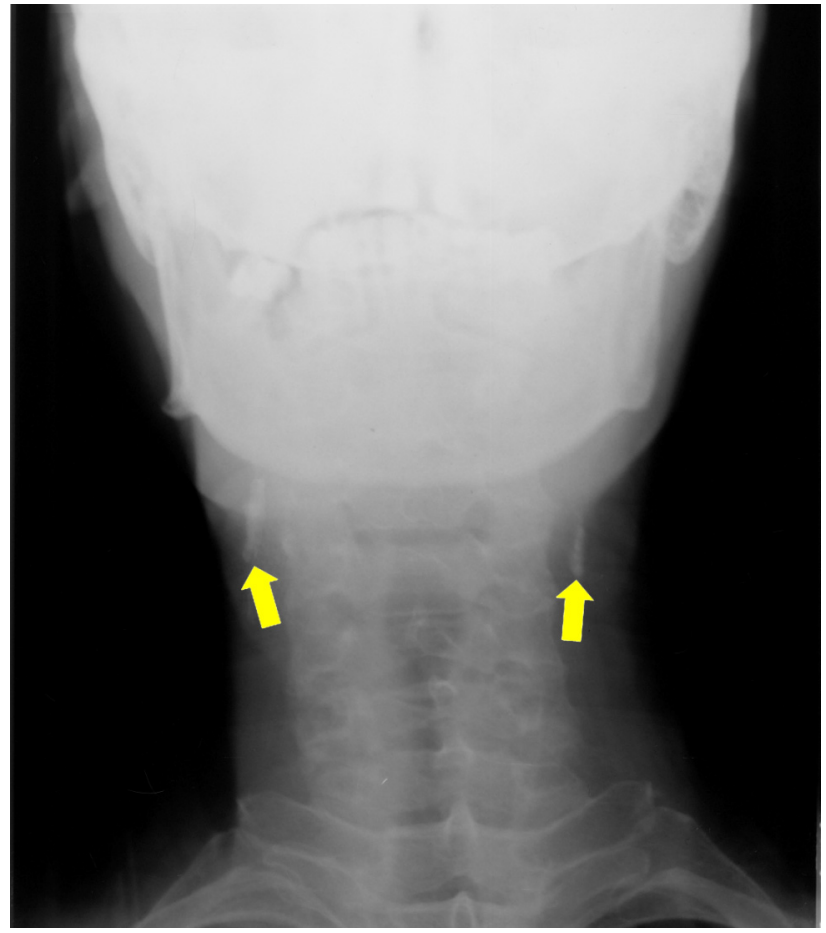

Fig. (3). Antero-posterior radiograph with arrows pointing out the carotid artery atheromas localized bilaterally.

Frankfurt plane parallel to the ground. This incidence, will allow visualization of the atheromas disposes laterally to the vertebrae, whereas the triticeal cartilages will practically not be observed, since their images will appear superimposed on the spinal column [27,28] (Fig. 3).

The precision of the panoramic radiograph in detecting carotid artery atheromas has been continually tested. In this context, was evaluated by a cardiologist the prevalence of calcification and stenosis of the carotid arteries in 104 patients who for various reasons, underwent Doppler ultrasonography. The same patients were submitted to panoramic radiographs, and the conclusion of the study was that due to low sensitivity and positive predictive value found, the panoramic cannot be considered an accurate or reliable test for calcification or stenosis [26]. On the other hand, from another sample consisting of 83 panoramic radiographs, two experienced evaluators selected 32 exams with probable carotid artery atheromas. Ultrasonography with Doppler confirmed the existence of atheromas in 29 exams (90\%) selected by these evaluators, showing that by means of accurate diagnostic evaluation it is perfectly possible to obtain positive results with the use of panoramic radiography $[25,29]$.

In spite of the value of panoramic radiography in the auxiliary diagnosis of carotid artery atheromas, there are more specific imaging exams indicated for this purpose. The thermograph and computerized tomography for example, are two imaging methods that provide the real extension and localization of calcifications with precision, as well as the degree of obliteration of the carotid artery involved. Whereas ultrasonography with Doppler, considered the gold standard, in addition to providing all the precision of the two abovementioned methods, has the enormous advantage of being a noninvasive and inexpensive method, with very low morbidity [29-31].

\section{DISCUSSION}

Atherosclerosis is the main cause of myocardial infarctions and strokes in the world population, determining the death of thousands of persons. The disease presents a complex etiopathogenesis involving a sequence of biological mechanisms that are influenced by various factors $[1,2,4]$. Diabetes mellitus, obesity, arterial hypertension and inadequate diet and eating habits are the classical risk factors for this disease, and by means of new researches, other factors have been suggested as being favorable to its development, such as, for example, chronic renal disease, menopause, radiotherapy of the head and neck, and the obstructive sleep apnea syndrome $[5,20]$.

Atheromas are the base plates of atherosclerosis, especially constituted of lipids and fibrous tissue, which are deposited on the arterial walls and become calcified, making it feasible to identify them in certain radiographic exams. In view of this, the atheromas that affect the carotid arteries are capable of being viewed in panoramic radiograph, widely used exam in routine dental practice [3, 21-30]. The diagnosis of atheromas in panoramic radiographs may not be so simple, as various other entities may be present in the same region. The triticeal cartilage, when calcified, is no doubt the main anatomic finding to be considered in the differential diagnosis and imaging evaluation must preferably be performed by an experienced radiologist who can eventually make use of an antero-posterior radiograph when faced with doubtful diagnoses [27, 28].

The most indicated exams for identifying atheromas are ultrasonography with Doppler and computerized tomography, because of the richness of diagnostic information they provide, however, the enormous use of the panoramic exam, especially in dentistry, makes this radiograph an auxiliary important tool in the diagnosis of carotid artery atheromas and the dentist, the professional of choice for this evaluation [24, 25, 29].

Atherosclerosis is a cardiovascular disease with enormous impact all over the world, and preventive measures must be taken to diminish its incidence. Changes in lifestyle, including adequate diet and eating habits, practicing physical activity and a less stressful life are important strategies in the primary prevention of this pathology. When dentists are faced with a suspicion of the presence of carotid artery atheromas in panoramic radiographs, they play a important role in guiding and immediately referring their patients to doctors for adequate medical treatment, thus contributing to many lives possibly being saved.

\section{CONCLUSIONS}

- Atherosclerosis is a disease with a high incidence all over the world and the main factor responsible for strokes and myocardial infarctions.

- There is a series of risk factors that favor the development of the disease, such as: diabetes mellitus, obesity, arterial hypertension, inadequate diet and eating habits. Adopting habits of a healthy lifestyle and 
knowing the risk factors are essential measures to be practiced by the population to combat atherosclerosis.

- Although it may not be the exam of choice, panoramic radiography can identify carotid artery atheromas and once they have been identified, the dentist must inform and instruct patients to seek medical evaluation as soon as possible.

- The identification of carotid artery atheromas in panoramic radiographs must preferably be performed by an experienced evaluator, and the triticeal cartilage is the most important anatomic structure in the differential diagnosis.

\section{ACKNOWLEDGEMENTS}

We would like to thank Dr. Jefferson Luis Oshiro Tanaka, for his valuable contribution in drawing up the illustrative diagrams in Fig. (1 and $\mathbf{2}$ ). We are equally grateful to Dr. Maurício Anderson Fernandes, for providing the panoramic radiograph presented in Fig. (2) and the anteroposterior radiograph shown in Fig. (3).

\section{REFERENCES}

[1] Zhdanov VS, Sternby NH. Monitoring of atherosclerosis. Int J Cardiol 2004; 95: 39-42.

[2] Fatahzadeh M, Glick M. Stroke:epidemiology, classification, risk factors, complications, diagnosis, prevention, and medical and management. Oral Surg Oral Med Oral Pathol Oral Radiol Endod 2006;102:180-91.

[3] Kamikawa RS, Pereira MF, Fernandes A, Meurer MI. Study of the localization of radiopacities similar to calcified carotid atheroma by means of panoramic radiography. Oral Surg Oral Med Oral Pathol Oral Radio Endod 2006; 101: 374-8.

[4] Hansson GK. Atherosclerosis- An immune disease. Atherosclerosis 2009; 202: 2-10.

[5] Matthews DC, Perio D. The relationship between diabetes and periodontal disease. J Can Dent Assoc 2002; 68: 161-4.

[6] Friedlander AH, Garret NR, Norman DC. The prevalence of calcified carotid artery atheromas on the panoramic radiography of patients with type 2 diabetes mellitus. J Am Dent Assoc 2002; 133: 1516-23.

[7] Cole TJ, Bellizzi MC, Flegal km, Dietz WH. Establishing a standard definition for child overweight and obesity worldwide: int survey. Br Med J 2000; 320: 1-6.

[8] Trepels T, Zeiher AM, Fichtlscherer S. The endothelium and inflammation. Endothelium 2006;13: 423-9.

[9] Strahan EJ, White K, Fong GT, Fabrigar LR, Zanna MP, Cameron R. Enhancing the effectiveness of tobacco package warning labels: a social psychological perspective. Tob Control 2002; 11: 183-90.

[10] Lewis MJ, Wackowski O. Dealing with an innovative industry: a look at flavored cigarettes promoted by mainstream brands. Am J Public Health 2006; 96: 244-5.

[11] Damiani IT, Gagliardi RJ, Scaff M. The influence of the ethanol in alcoholic beverages in the extracranial carotid arteries atherosclerosis. Arq Neuropsiquiatr 2004; 62:1022-6.

[12] Kang JX, Leaf A. Prevention of fatal cardiac arrhythmias by polyunsaturated fatty acids. Am J Clin Nutr 2000; 71: 202-7.

[13] Kansu O, Ozbek M, Avcu N, Gençtoy G, Kansu H, Turgan C. The prevalence of carotid artery calcification on the panoramic radiographs of patients with renal disease. Dentomaxilofac Radiol $2005 ; 34: 16-9$.

[14] Alevizaki M, Saltiki K, Cimponeriu A, et al. Severity of cardiovascular disease in postmenopausal women: associations with common estrogen receptor alpha polymorphic variants. Eur J Endocrinol 2007; 156: 489-96.

[15] Friedlander AH, Altman L. Carotid artery atheromas in postmenopausal women. J Am Dental Assoc 2001; 132:1130-6.

[16] Panico MDB. Hiper-homocisteinemia e doença vascular. J Vas $\mathrm{Br}$ 2004; 3: 3-4.

[17] Freymiller EG, Sung EC, Friedlander AH. Detection of radiationinduced cervical atheromas by panoramic radiography. Oral Oncol 2000; 36: 175-9.

[18] Bauer M, Mohlenkamp S, Lehmann N, et al. The effect of age and risk factors on coronary and carotid artery atherosclerotic burden in males. Atherosclerosis. 2009; 205: 595-602.

[19] Williamson DA, Rejeski J, Lang W, Van Dorsten B, Fabricatore AN, Toledo K. Impact of a weight management program on healthrelated quality of life in overweight adults with type 2 diabetes. Arch Intern Med 2009; 169: 163-71.

[20] Gottlieb MGV, Bonardi G, Moriguchi EH. Physiopatology and inflammatory aspects of atherosclerosis. Sci Med 2005; 15: 203-7.

[21] Ardakani FE, Ardakani MA, Mohammadi Z, Sheikhha MH. Evaluating calcified carotid artery atheromas in panoramic radiographs of patients with type 2 diabetes mellitus. Oral Radiol 2007; 23: 6-9.

[22] Friedlander AH, Cohen SN. Panoramic radiographic atheromas portend adverse vascular events. Oral Surg Oral Med Oral Pathol Oral Radiol Endod 2007; 103: 830-5.

[23] Griniatsos J, Damaskos S, Tsekouras N, Klonaris C, Georgopoulos S. Correlation of calcified carotid plaques detected by panoramic radiograph with risk factors for stroke development.Oral Surg Oral Med Oral Pathol Oral Radiol Endod 2009;108: 600-03.

[24] Madden RP, Hodges JS, Salmen CW, et al. Utility of panoramic radiographs in detecting cervical calcified carotid atheroma. Oral Surg Oral Med Oral Pathol Oral Radiol Endod 2007; 103: 543-8.

[25] Almog DM, Tsimidis K, Moss ME, Gottlieb RH, Carter LC. Evaluation of a training program for detection of carotid artery calcifications on panoramic radiographs. Oral Surg Oral Med Oral Pathol Oral Radiol Endod 2000; 90: 111-7.

[26] Manzi FR, Guedes FR, Duarte RS, Tureli MCM, Almeida SM, Bóscolo FN. Identificação de pacientes com risco de derrame na clínica odontológica por meio de radiografias panorâmicas. Rev Bras Odontol 2005; 62: 238-40.

[27] Carter LC. Discrimination between calcified triticeous cartilage and calcified carotid atheroma on panoramic radiography. Oral Surg Oral Med Oral Pathol Oral Radio Endod 2000; 90:108-10.

[28] Ahmad M, Madden R, Perez L. Triticeous cartilage: prevalence on panoramic radiographs and diagnostic criteria. Oral Surg Oral Med Oral Pathol Oral Radiol Endod 2005; 99: 225-30.

[29] Ravon NA, Hollender LG, McDonald V, Persson GR. Signs of carotid calcification from dental panoramic radiographs are in agreement with Doppler sonography results. J Clin Periodontol 2003; 30: 1084-90.

[30] Pornprasertsuk-Damrongsri S, Virayavanich W, Thanakun S, Siriwongpairat P, Amaekchok P, Khovidhunkit W. The prevalence of carotid artery calcifications detected on panoramic radiographs in patients with metabolic syndrome. Oral Surg Oral Med Oral Pathol Oral Radiol Endod 2009; 108: 57-62.

[31] Tanaka T, Morimoto Y, Ansai T, et al. The presence of carotid artery calcification on panoramic radiographs predict the risk of vascular diseases among 80-year-olds? Oral Surg Oral Med Oral Pathol Oral Radiol Endod 2006; 101: 777-83.

(C) Henriques et al.; Licensee Bentham Open.

This is an open access article licensed under the terms of the Creative Commons Attribution Non-Commercial License (http://creativecommons.org/licenses/by-nc/3.0/) which permits unrestricted, non-commercial use, distribution and reproduction in any medium, provided the work is properly cited. 